\title{
Determinação dos Parâmetros de um Filtro Adaptativo utilizado como Ferramenta para Controle de Qualidade em Linha de Montagem de Câmbios Automotivos usando Evolução Diferencial
}

\section{Determination of Parameters of an Adaptive Filter used as Quality Control Tool in Automotive Gearbox Mounting Line using Differential Evolution}

Ricardo Humberto de Oliveira Filho ${ }^{1}$; Fran Sérgio Lobato²

\section{RESUMO}

As tradicionais técnicas empregadas para a análise de sinais, em conjunto com o monitoramento de vibrações oriundas do funcionamento de câmbios automotivos, podem ser utilizadas como metodologia em programas de controle de qualidade. Todavia, devido à complexidade da dinâmica de destes sistemas e aos ruídos inerentes a uma linha de produção, existe uma grande dificuldade na definição dos sintomas de controle e determinação de valores limites. Neste sentido, este trabalho tem por objetivo empregar o algoritmo de Evolução Diferencial para a determinação dos parâmetros de um filtro adaptativo empregado como ferramenta para avaliar o controle de qualidade em uma linha de montagem de câmbios via monitoramento de vibrações. Para a avaliação da metodologia proposta foram utilizados sinais sintéticos para representam o engrenamento e possíveis defeitos existentes em seus componentes. Os resultados obtidos demostraram que o otimizador foi capaz de filtrar os sinais relacionados ao engrenamento e ao resíduo proveniente de falhas de fabricação ou montagem.

Palavras-chave: Câmbios Automotivos, Filtro Adaptativo, Evolução Diferencial.

\section{ABSTRACT}

The traditional techniques used for the analysis of signals, associated to monitoring of vibrations from the operation of automotive gearbox, can be used as methodology in quality control programs. However, due to complexity of dynamics of these systems and to noise inherent in production line, there is great difficulty in defining the control symptoms and the determination of limit values. In this context, this work aims to use the Differential Evolution algorithm to determinate of parameters of an adaptive filter used as tool to evaluate the quality control during the monitoring of vibrations in automotive gearbox. To evaluate the proposed methodology, synthetic signals considered to represent the gear and possible defects in its components are used. The results showed that the optimizer was able to filter the signals related to the gear and the residue from manufacturing or assembly failures.

Keywords: Automotive Gearbox, Adaptive Filter, Differential Evolution. 


\section{INTRODUÇAOO}

Nos últimos anos, várias pesquisas têm sido desenvolvidas com o intuito de aumentar a vida útil das caixas de transmissão em automóveis. Dentre as diversas vertentes que podem ser encontradas, o estudo dos fatores pertinentes ao projeto, tais como os efeitos decorrentes da geometria inadequada dos dentes das engrenagens, é uma das mais importantes (HOWARD et al., 2001; VELEX e MAATAR, 1996; AMABILI e FREGOLENT, 1998). Além disso, há que se destacar as pesquisas envolvendo a análise dos sinais, também conhecido como análise de assinaturas (BRAUN, 1986). Neste caso, objetiva-se predizer a vida útil das caixas de engrenagens (WANG e MCFADDEN, 1995; WANG et al., 2001; CHOl et al., 1996).

Neste contexto, Menegatti (1999) e Santana et al. (1998) desenvolveram uma série de procedimentos computacionais e experimentais utilizados como ferramenta de apoio na quantificação e identificação das possíveis fontes de rumorosidade nas caixas de transmissão. Foram realizados ensaios em câmbios da unidade FIASA em Betim, Minas Gerais. Neste mesmo cenário, encontra-se em funcionamento um aplicativo que executa um procedimento "passa não passa", para a análise de curvas de mérito determinadas a partir de um banco de dados em câmbios para análise da manobrabilidade de máquinas (PENA, 2003). A partir destes testes pode-se observar que a rejeição ou não dos câmbios ensaiados, ao se utilizar o procedimento adotado, está associada a algum problema ocorrido durante o processo de fabricação ou montagem. Atualmente, constatada a rumorosidade, a definição das causas que resultaram em tal problema é efetuada por meio de testes dimensionais de todas as engrenagens, eixos e carcaças que compõem os câmbios. Considerando que a caracterização completa de uma engrenagem implica em dezenas de procedimentos metrológicos, conclui-se que a identificação da causa da rumorosidade tem custo elevado além de grande tempo despendido (PENA, 2003).

Um dos problemas observados durante a realização de manutenção preditiva por análise de vibrações é a dificuldade de aquisição dos sinais. Neste caso, os sinais medidos estão sujeitos a problemas relacionados à propagação do fenômeno vibratório pela máquina, devido à própria impedância mecânica da estrutura, que acarreta uma perda da energia oscilatória e pela atenuação da transmissão de forças de oscilação (MENEGATTI, 1999). Sabe-se também que o sinal obtido é contaminado por outras informações que não dizem respeito à detecção e diagnóstico de falhas em um determinado componente, como aquelas advindas de outras fontes vibratórias, além de ruídos externos diversos. Como 
tentativa de contornar este problema, uma ferramenta empregada é a filtragem dos sinais, permitindo desta forma uma análise mais confiável (MACÁRIO, 2006).

Neste contexto, surge naturalmente a necessidade do desenvolvimento de ferramentas que auxiliem os procedimentos de análise de sinais de vibrações e ruído. Para isto, pode-se propor a elaboração de uma ferramenta que realize a filtragem dos sinais de vibração provenientes de câmbios automotivos, extraindo as interferências que não sejam relacionadas ao bom funcionamento do câmbio, para que então se consiga avaliar os defeitos e falhas provenientes dos processos de fabricação e montagem de maneira mais criteriosa. Contudo, para uma filtragem adequada (obtenção de resultados confiáveis) é necessário que os parâmetros do filtro adaptativo sejam escolhidos de forma a se obter o menor erro possível entre os valores dos sinais estimados e experimentais.

Diante do que foi apresentado, o presente trabalho tem por objetivo a determinação dos parâmetros de um filtro adaptativo com algoritmo LMS (Least Mean Square) via formulação e resolução de um problema inverso. Para essa finalidade, considera-se o algoritmo de Evolução Diferencial (ED) como ferramenta de otimização. Este trabalho está estruturado como segue: a seção 2 apresenta uma breve revisão sobre aspectos relacionais a filtragem de sinais, a modelagem matemática do problema de otimização e a descrição do algoritmo de ED; Já na seção 3 é apresentada a descrição da metodologia proposta neste trabalho. Na seção 4 é apresentada os resultados obtidos com a aplicação da metodologia proposta. Finalmente, as conclusões são apresentadas na última seção.

\section{ESTADO DA ARTE}

\subsection{Filtragem Adaptativa}

Em relação às técnicas tradicionais de filtragem de sinais, a adaptativa é mais vantajosa quando há a necessidade de processar sinais cujas propriedades estatísticas do seu ambiente são inicialmente desconhecidas (MARQUES, 2003). Desta forma, estes filtros se adaptam de forma recursiva ao fenômeno que dá origem ao sinal mediante a variação de parâmetros da sua função de transferência a cada iteração, acompanhando as variações estatísticas dos sinais e, permitindo uma filtragem eficaz em casos em que as técnicas do processamento digital de sinais tradicional são deficitárias (MACÁRIO, 2006).

No caso de ambientes estacionários, as propriedades estatísticas podem ser avaliadas utilizando-se o filtro de Wiener, cujos parâmetros referentes a sua função de transferência são constantes e definidos sob a regra da minimização do erro médio 
quadrático entre a sua saída e uma resposta definida como desejada (OLIVEIRA FILHO, 2007). Primeiramente, são definidas condições iniciais, devido ao desconhecimento das propriedades estatísticas do sinal e, após um número de iterações, os parâmetros do filtro convergem para um conjunto conhecido como solução ótima de Wiener (OLIVEIRA FILHO, 2007).

Os filtros adaptativos também se destinam a extrair informações de interesse de um ambiente contaminado por uma interferência (ou ruído). Segundo Marques (2003), estes são lineares no sentido de que a saída oferecida é uma função linear das observações apresentadas à sua entrada.

A Figura 1 ilustra um diagrama no qual está representado um filtro adaptativo com seus componentes.

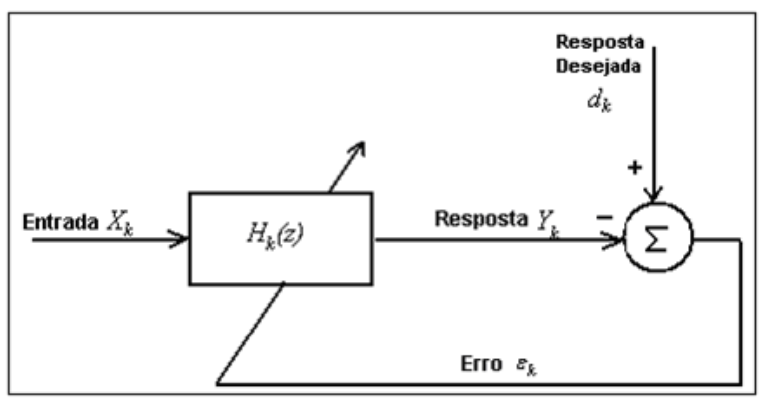

Figura 1: Diagrama representativo de um filtro adaptativo.

Fonte: OLIVEIRA FILHO, 2007.

Na Figura 1, $X_{k}$ é o sinal de entrada do filtro, $Y_{k}$ é a saída do filtro, $d_{k}$ é a saída desejada e $H_{k}(z)$ é a Função Transferência do filtro que sofrerá modificações em seus parâmetros via minimização do erro, dado pela Equação 1.

$$
\varepsilon_{k}=d_{k}-Y_{k}
$$

A mudança (ou adaptação) dos parâmetros do filtro e a busca pelo valor ótimo (erro mínimo) ficam a cargo dos algoritmos de adaptação. A escolha por um outro dependem de alguns fatores, dentre os quais pode-se citar (MARQUES, 2003): a velocidade de convergência (número de iterações para alcançar o objetivo), a precisão, a complexidade do problema a ser revolvido, e da estabilidade do algoritmo (possibilidade ou não de instabilidade numérica do algoritmo, motivada pelo acúmulo de erros provenientes da precisão finita do mesmo). 
Apesar da grande quantidade, os algoritmos de adaptação dividem-se em duas grandes classes: os que se baseiam no gradiente e os que seguem os mínimos quadrados do sinal do erro. Os algoritmos de gradiente baseiam-se no filtro de Wiener, estimando o gradiente da superfície das funções de custo. Já os algoritmos de mínimos quadrados são baseados no filtro de Kalman, onde de forma determinística, minimizam a soma dos quadrados dos erros parciais (NUNES, 2006).

Para aplicação da técnica de cancelamento adaptativo de ruído, técnica a qual será descrita posteriormente e utilizada neste trabalho, é vantajoso o uso do algoritmo do gradiente, mais conhecido como LMS - Least Mean Square, devido ao seu baixo custo computacional e ao melhor comportamento que este exibe no seguimento das não estacionaridades.

\subsection{O Problema de Otimização}

O problema de otimização consiste na determinação do vetor de variáveis de projeto que minimizem (ou maximizem) o valor de uma função objetivo sujeita ou não a restrições algébricas de igualdade e/ou desigualdade, equações diferenciais ordinárias e/ou parciais e a restrições laterais (domínio ou espaço de busca das variáveis de projeto).

Matematicamente, o problema algébrico geral de otimização pode ser descrito pela Equação 2 (VANDERPLAATS, 1999).

$$
\left\{\begin{array}{l}
\min F(X), X=\left[X_{1}, X_{2}, \ldots, X_{n}\right]^{T} \operatorname{com} X \in \square^{n} \\
h_{k}(X)=0, k=1,2, \ldots, K \\
g_{j}(X) \leq 0, j=1,2, \ldots, J \\
X_{i}^{L} \leq X \leq X_{i}^{U}, i=1,2, \ldots, n
\end{array}\right.
$$

em que $X$ é o vetor de variáveis de projeto, $F(X)$ é a função a ser otimizada, $g$ e $h$ representam os vetores de restrições de desigualdade e igualdade, respectivamente, e $X^{L}$ e $X^{U}$ os limites inferior e superior para o vetor de variáveis de projeto.

De forma geral, os métodos existentes para a resolução dos problemas de otimização podem ser divididos em Clássicos (ou Determinísticos) e Não Clássicos (ou Não Determinísticos ou Aleatórios).

As Determinísticas são fundamentadas no uso de informações sobre o gradiente da função objetivo e das restrições para a atualização de um ponto de partida (estimativa inicial). Sua aplicabilidade se deve, principalmente, à sofisticação dos recursos numéricos e computacionais observado nas últimas décadas. Estas técnicas podem apresentar 
dificuldades numéricas tais como: dedicam todo o seu esforço em uma única estimativa inicial; observa-se problemas relacionados à falta de continuidade das funções ou de suas restrições, bem como a presença de funções não convexas; a existência de ruídos nas funções; a necessidade de se trabalhar com valores discretos para as variáveis; e a existência de mínimos ou máximos locais (VANDERPLAATS, 1999; EDGAR et al., 2001; SARAMAGO, 2003).

Já as técnicas Não-Determinísticas são baseadas nos processos de seleção natural da luta pela vida, da genética de populações ou em estratégias puramente estruturais. $O$ desenvolvimento dessas metodologias pseudoaleatórias se deu por volta de $1950 \mathrm{com}$ o surgimento dos Algoritmos Genéticos, quando biólogos utilizavam técnicas computacionais para a simulação de sistemas observados na natureza. Mas foi somente entre 1960 e 1970, na Universidade de Michigan sob a direção de John Holland em 1975, que iniciou o estudo de algoritmos genéticos como os conhecidos atualmente.

Atualmente, deve-se destacar a diversidade da concepção conceitual com que essas metodologias são fundamentadas. Dentre os mais conhecidos e aplicados na literatura, pode-se citar, além dos Algoritmos Genéticos, o Recozimento Simulado, o Enxame de Partículas, a Colônia de Formigas, o Sistema Imunológico, os Modelos de Ciclo de Vida e a Evolução Diferencial.

\subsection{Evolução Diferencial}

O algoritmo de ED é uma estratégia de otimização estrutural proposta por Storn e Price (1995) para a resolução de problemas de otimização com um único objetivo. Em linhas gerais, este é baseado na geração de candidatos em potencial a partir da aplicação de operações vetoriais. Seu procedimento geral é dado pelas seguintes etapas (STORN \& PRICE, 1995):

- Inicialmente, gera-se, aleatoriamente, uma população com NP indivíduos considerando os limites das variáveis de projeto definidos pelo usuário;

-Seleciona-se três indivíduos, de forma aleatória, um para ser substituído $\left(x_{1}\right)$ e os outros dois $\left(x_{2}\right.$ e $\left.x_{3}\right)$ para realizar o a subtração vetorial;

- Aplica-se a subtração vetorial considerando os indivíduos $x_{2}$ e $x_{3}$. A esse vetor resultante multiplica-se um escalar $F$, definido como taxa de perturbação. Esse vetor 
é somado ao vetor $X_{1}$ para gerar um candidato em potencial $\left(X_{\text {new }}\right)$. Matematicamente, este processo é dado pela Equação 3.

$$
x_{\text {new }}=X_{1}+F\left(x_{2}-x_{3}\right)
$$

Esse procedimento é repetido até que uma nova população com NP candidatos seja gerada.

- Cada novo candidato poderá substituir o correspondente (em termos de posição) se for gerado um número aleatório entre zero e um e este for menor do que uma probabilidade de cruzamento $(C R)$, também definido pelo usuário. Assim, ao final de uma geração, a população corrente será formada por candidatos pertencentes a população original e a nova população;

- Este procedimento é repetido até que o número total de gerações (ou outro critério adotado) seja satisfeito.

O algoritmo proposto por Storn e Price (1995) possibilita ao usuário a escolha de diferentes estratégias de cruzamento (ver a Equação 3). A Tabela 1 apresenta as estratégias propostas por estes autores.

Tabela 1. Estratégias de cruzamento propostas por Storn e Price (1995).

\begin{tabular}{l|l}
\hline $1-\mathrm{DE} /$ best/1/exp & $6-\mathrm{DE} / \mathrm{best} / 1 /$ bin \\
$2-\mathrm{DE} / \mathrm{rand} / 1 / \exp$ & $7-\mathrm{DE} / \mathrm{rand} / 1 /$ bin \\
$3-\mathrm{DE} /$ rand-to-best/1/exp & $8-\mathrm{DE} / \mathrm{rand}$-to-best/1/bin \\
$4-\mathrm{DE} /$ best/2/exp & $9-\mathrm{DE} /$ best/2/bin \\
$5-\mathrm{DE} / \mathrm{rand} / 2 / \exp$ & $10-\mathrm{DE} / \mathrm{rand} / 2 / \mathrm{bin}$
\end{tabular}

Fonte: Adaptado de Storn e Price (1995).

A convenção geral usada nesta tabela é $D E / x / y / z$. DE representa Evolução Diferencial (Differential Evolution), $x$ representa o vetor que será perturbado, $y$ é o número de vetores que são utilizados para a perturbação de $x$, e $z$ representa o tipo de cruzamento que será realizado (exp=exponencial ou bin=binomial).

A escolha dos valores dos parâmetros utilizados pelo algoritmo de ED (NP, F, CR e a estratégia de cruzamento) não é uma tarefa trivial. Todavia, normalmente adota-se $N P$ como sendo um valor que varia de 5 a 10 vezes o número de variáveis de projeto do problema em análise. $F$ é tomado como sendo um valor entre 0,4 a 2,0. Já $C R$ pode ser 
arbitrado como sendo igual a 0,9 (STORN \& PRICE, 1995). É importante ressaltar que estes valores podem variar para cada estudo de caso.

\section{METODOLOGIA}

Para a obtenção dos parâmetros do filtro, é necessário a formulação e a resolução de um problema inverso. Este consiste da determinação do vetor de variáveis de projeto de modo a minimizar a diferença entre os valores simulados e experimentais. Para essa finalidade, considera-se neste estudo o sinal obtido de um câmbio com defeito na quinta marcha, em que uma varredura senoidal (sine sweep) representará o sinal de engrenamento e uma parcela de harmônicos e de ruído branco representará os possíveis defeitos de fabricação e montagem. A varredura senoidal inicia-se em $800 \mathrm{~Hz}$ e, durante 1 segundo, aumenta linearmente até $3800 \mathrm{~Hz}$, mantendo-se constante neste valor durante 1 segundo. Em seguida, durante 1 segundo diminui linearmente para $1200 \mathrm{~Hz}$.

Na Figura 2 é ilustrado o espectrograma (Transformada Curta de Fourier, ou Short Fourier Transform) do sinal de engrenamento simulado. A codificação de cor na lateral da Figura 2 indica a amplitude do sinal em dB.

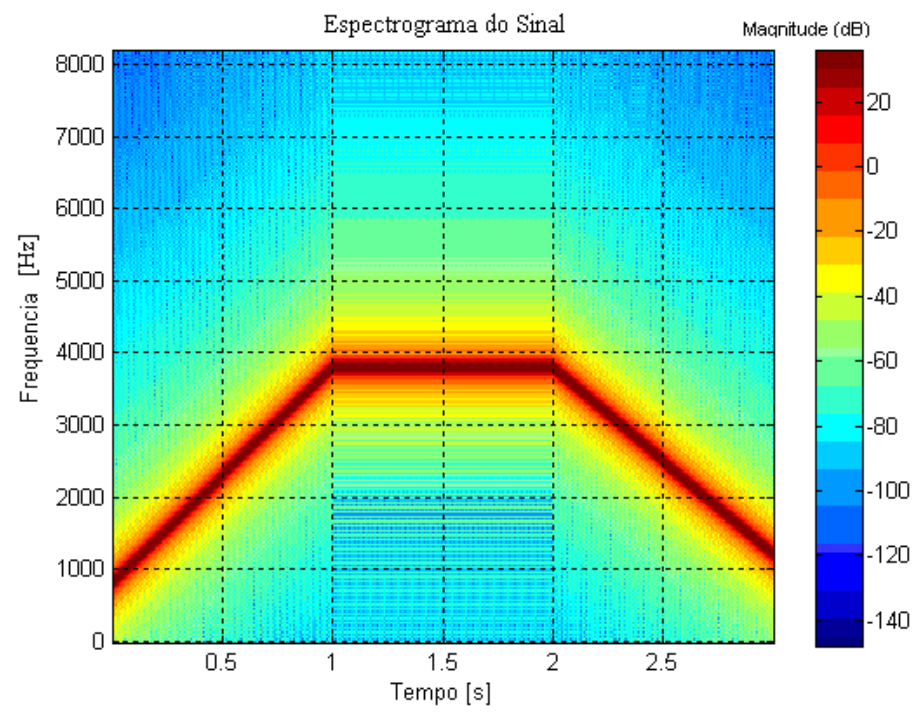

Figura 2: Espectrograma do sinal de engrenamento simulado.

Para simular os defeitos que possam ocorrer devido a erros de montagem e fabricação, será adicionado um sinal de defeito composto por um ruído branco com 50\% da potência da varredura senoidal, um harmônico com frequência de $2000 \mathrm{~Hz}$ e com 
defasagem de $60^{\circ}$ e $50 \%$ da potência da varredura senoidal, e um harmônico com frequência de $3000 \mathrm{~Hz}$ e com amplitude variável.

A Figura 3 ilustra a comparação entre o sinal de engrenamento isento de defeitos (Referência) e o sinal de engrenamento com os defeitos simulados (Primário).

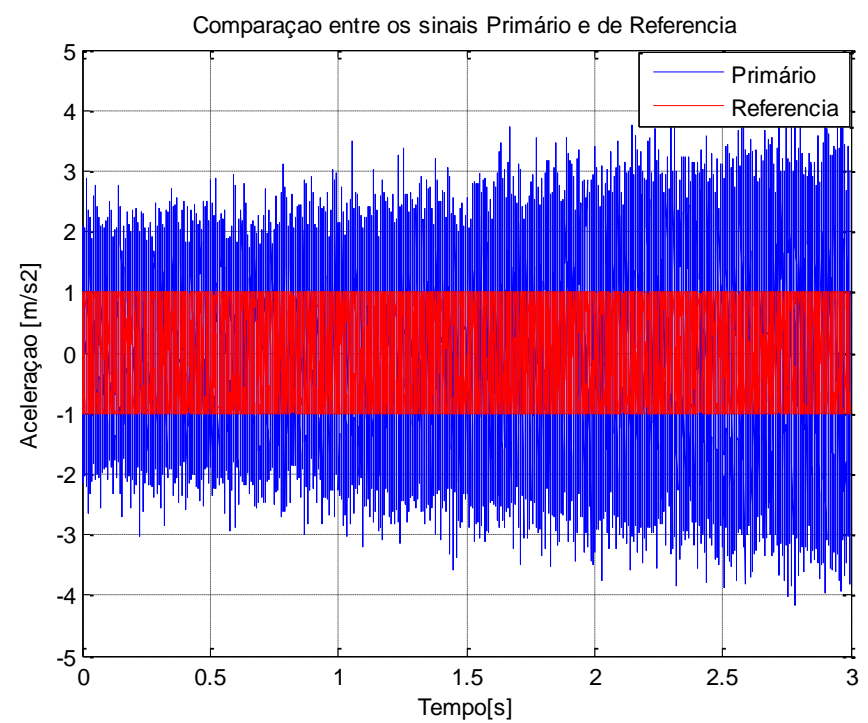

Figura 3: Comparação entre os sinais de engrenamento para os casos isento de defeitos (Sinal de Referência) e com os defeitos simulados (Sinal Primário).

$\mathrm{Na}$ análise de sinais de comportamento transiente e não estacionário, onde a frequência varia com o tempo, é necessário o uso de metodologias de análises tempofrequência. Neste trabalho foi utilizada a média exponencial $\left(\bar{x}_{i}\right)$, dada pela Equação 4.

$$
\bar{x}_{i}^{2}=x_{i-1}^{2}+\left(x_{i}^{2}-x_{i-1}^{2}\right) / k
$$

sendo $k$ dado pela Equação 5 .

$$
k=f_{\text {aquis }} T+1
$$

onde $f_{\text {aquis }}$ é a frequência de aquisição em $\mathrm{Hz}$ e $T$ é o tempo de integração, sendo este, neste trabalho, igual a $125 \times 10^{-3} \mathrm{~s}$, o que corresponde a um tempo de integração rápido, permitindo então um "esquecimento" das ocorrências passadas no sinal analisado.

Conhecendo as características do sinal em estudo, aplica-se a técnica da filtragem adaptativa utilizando um filtro adaptativo com algoritmo LMS normalizado de forma a obter um sinal de engrenamento sem a interferência das fontes externas. Na Figura 4 ilustra-se o diagrama de filtragem adaptativa utilizado nesta simulação, onde $d$ é o sinal de referência, representado pelo sinal de engrenamento simulado (varredura senoidal); $r$ é o ruído 
provindo de fontes externas ao funcionamento do câmbio (defeitos de fabricação e montagem); $x$ é o sinal primário, representado pelo sinal de engrenamento contaminado pelo ruído; o é o sinal filtrado; $y$ é o sinal de engrenamento estimado; e e é o erro ente os sinais filtrado e de referência.

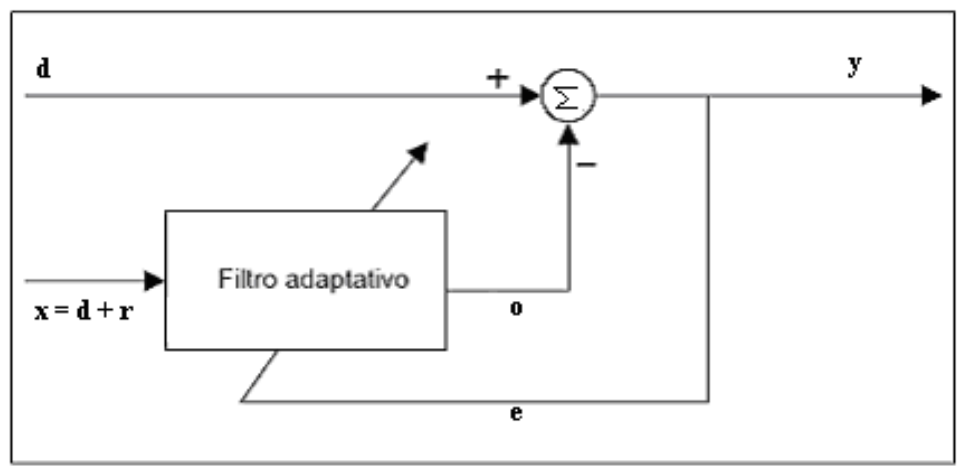

Figura 4: Diagrama da técnica da filtragem adaptativa aplicada ao trabalho.

Em resumo, deseja-se minimizar o erro quadrático médio a partir da determinação de $L \in\{1,2,3,4,5, \ldots, 50\} ; 0<\mu<1 ; 0,0001<\alpha<1$. Por se tratar de um problema com variáveis reais e inteiras, deve-se realizar uma pequena modificação no algoritmo de ED para que a variável $L$ possa ser determinada, visto que em sua concepção conceitual, esse algoritmo não é capaz de lidar com variáveis que não sejam contínuas.

Para essa finalidade, seja um problema com uma única variável de projeto $\left(X_{\text {disc }}\right)$ que assuma apenas valores discretos ( $x_{\text {disc }} \in\{1,242,453,094,675,87\}$ ). Inicialmente deve-se redefinir essa variável de projeto em um intervalo qualquer, como por exemplo, $0 \leq x_{\text {disc }} \leq$ 1. Dessa maneira, como se têm 5 valores discretos, pode-se escrever o critério de escolha conforme a Equação (7).

$$
x_{\text {disc }}= \begin{cases}1,24 & \text { se } 0,0 \leq x_{\text {cont }} \leq 0,2 \\ 2,45 & \text { se } 0,2<x_{\text {Cont }} \leq 0,4 \\ 3,09 & \text { se } 0,4<x_{\text {cont }} \leq 0,6 \\ 4,67 & \text { se } 0,6<x_{\text {cont }} \leq 0,8 \\ 5,87 & \text { se } 0,8<x_{\text {cont }} \leq 1,0\end{cases}
$$

Assim, se um candidato apresentar um valor contínuo escolhido como sendo 0,564, esse valor é corrigido para 3,09 (que seria o correspondente no domínio discreto para essa variável contínua). O mesmo raciocínio pode ser utilizado para variáveis inteiras e binárias. Com esta simples mudança no algoritmo de ED, problemas que não apresentam somente variáveis contínuas podem ser tratados. 
Para fins de comparação dos resultados obtidos com a metodologia proposta, serão realizadas simulações para a determinação da melhor configuração considerando pares de combinações entre as variáveis de projeto. Neste contexto, foram gerados vetores com possíveis valores para os coeficientes $L=\{1,2,3,4,5, \ldots, 20\} ; \mu=0: 0,001: 1$ e $\alpha=0: 0,001: 1$. Para cada combinação, calcula-se o erro quadrático médio.

\section{RESULTADOS E DISCUSSAOO}

A Tabela 2 apresenta os resultados obtidos com a aplicação do algoritmo de ED considerando 10 corridas independentes e os seguintes parâmetros: 10 indivíduos; 100 gerações; taxa de perturbação e probabilidade de cruzamento iguais a 0,8 ; respectivamente e estratégia 7 (DE/rand/1/bin).

Tabela 2. Resultados obtidos pelo algoritmo de ED.

\begin{tabular}{ccccc}
\hline \multirow{2}{*}{ Execução } & \multicolumn{3}{c}{ Variáveis de Projeto } & \multirow{2}{*}{ Erro Quadrático Médio $(\mathrm{dB})$} \\
& $L$ & $\mu$ & $\alpha$ & 0,000797 \\
2 & 1 & 0,00084 & 0,53617 & 0,000797 \\
3 & 1 & 0,00084 & 0,53617 & 0,000587 \\
4 & 2 & 0,00080 & 0,65013 & 0,000797 \\
5 & 1 & 0,00084 & 0,53617 & 0,000797 \\
6 & 1 & 0,00084 & 0,53617 & 0,000797 \\
7 & 1 & 0,00084 & 0,53617 & 0,001274 \\
8 & 4 & 0,00083 & 0,05968 & 0,000797 \\
9 & 1 & 0,00084 & 0,53617 & 0,001821 \\
10 & 15 & 0,00201 & 0,31695 & 0,000797
\end{tabular}

Analisando os resultados da Tabela 2 constata-se que a função objetivo assume maiores valores para os maiores valores para os parâmetros $L$ e $\mu$ (15 e 0,00201; respectivamente).

Assim, o melhor resultado obtido pelo algoritmo de ED foi o conjunto [ $L \mu \alpha$ erro]=[2 $0,000800,650130,000587]$. Em relação ao obtido via simulação considerando diferentes pares, obteve-se [ $L \mu \alpha$ erro]=[2 0,001 0,05 0,000736]. Este valor, conforme esperado, é superior ao obtido pelo algoritmo de ED, visto que o mesmo não foi obtido considerando 
uma ferramenta de otimização. Assim, observa-se que o resultado obtido pelo algoritmo de ED é $20,25 \%$ menor do que o obtido pela simulação.

A Figura 5 apresenta a comparação entre os sinais estimados com o filtro utilizando os parâmetros otimizados nas corridas 1, 3, 7 e 9, com os sinais primário e de referência. Nesta figura percebe-se uma boa concordância entre os valores simulados com os parâmetros obtidos com o algoritmo de ED e o sinal de referência.

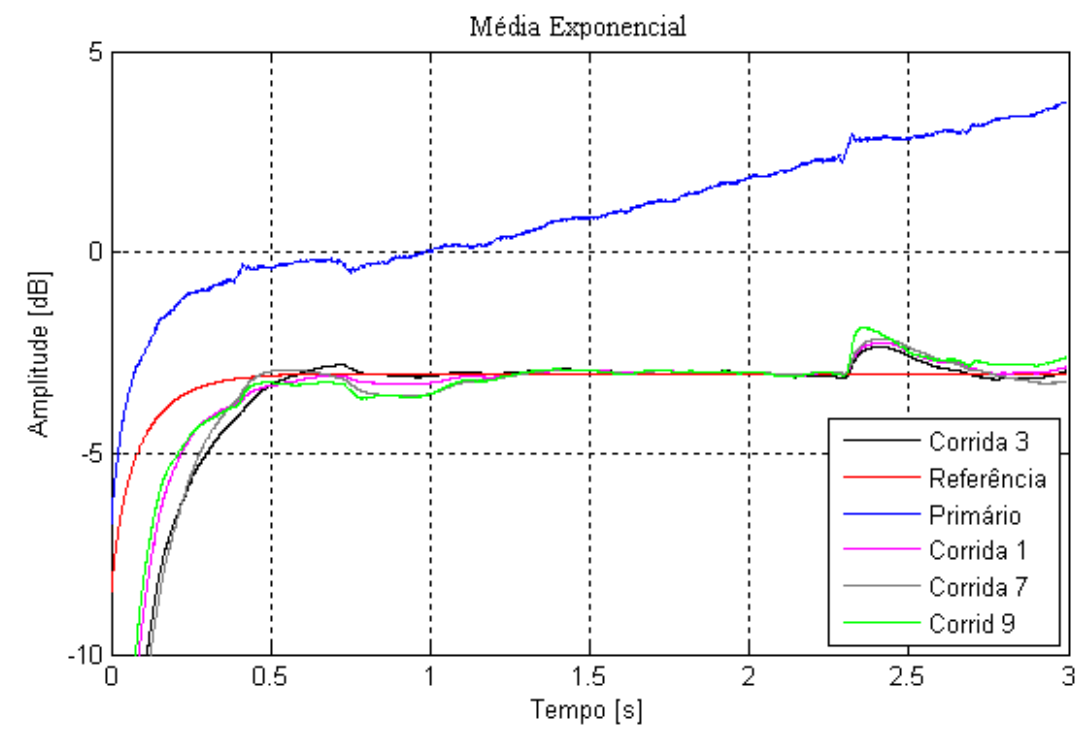

Figura 5: Comparação entre os sinais estimados pelo procedimento de otimização via ED.

Na Figura 6 é apresentado é apresentado um zoom a partir de 0,56 segundos.

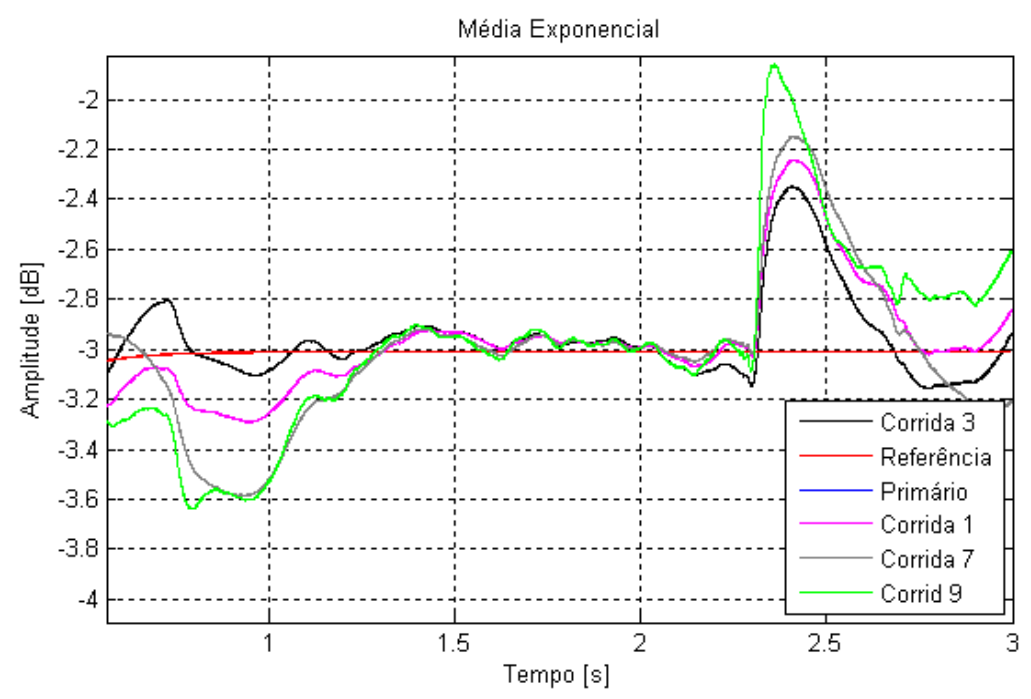

Figura 6: Destaque (zoom) da parte dos sinais estimados onde se verifica convergência. 
A Figura 7 apresenta a comparação entre as curvas obtidas com os parâmetros via simulação (denominada de Metodologia 1, isto é; para [ $L \mu \alpha$ erro]=[2 0,001 0,05 0,000736]) e considerando o algoritmo de ED ([L $\mu \alpha$ erro] $]=[20,00080$ 0,65013 0,000587]).

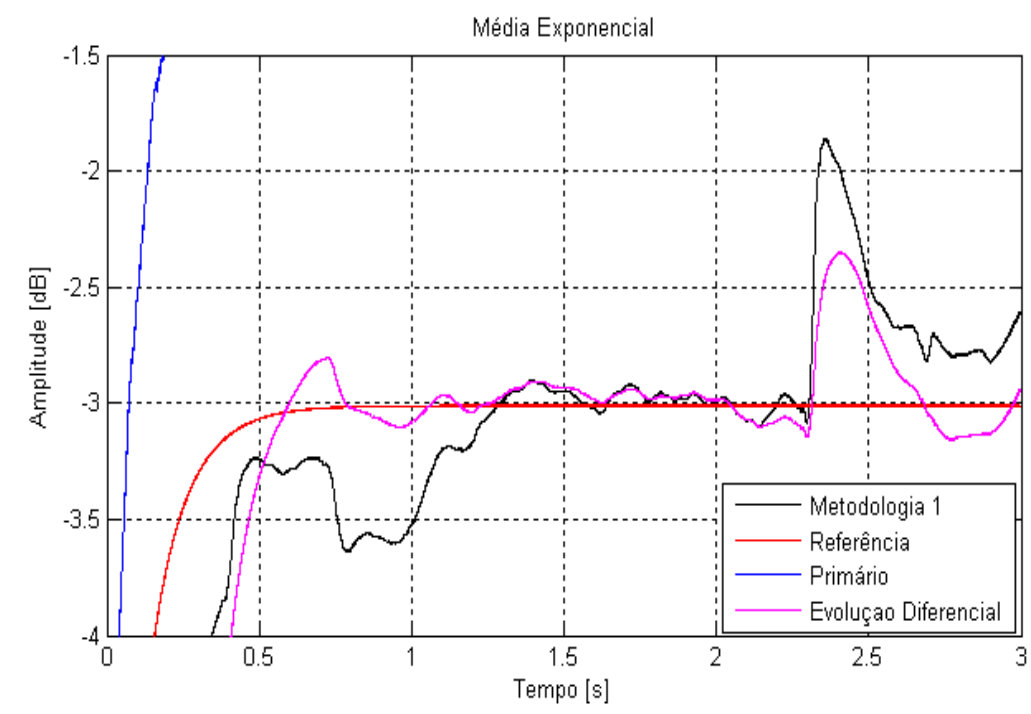

Figura 7: Média Exponencial dos sinais primário, referência e estimado.

\section{CONCLUSOES}

No presente trabalho foi desenvolvido um filtro adaptativo LMS (Least Mean Square) para ser empregado como ferramenta para controle de qualidade em uma linha de fabricação e montagem de câmbios automotivos. Para essa finalidade simulou-se um sinal de engrenamento com defeitos de fabricação e montagem, bem como aplicou-se o algoritmo de ED como ferramenta de otimização.

A partir dos resultados obtidos foi possível concluir que a metodologia proposta foi capaz de obter um resultado cerca de $21 \%$ melhor do que o simulado. Com a utilização do filtro adaptativo LMS associado ao algoritmo de ED foi possível obter um conjunto de parâmetros que minimizou a diferença entre os sinais simulado e de referência, não importando a quantidade ou intensidade de defeitos presentes no sinal primário (com ou sem modulações em amplitude ou em fase).

\section{AGRADECIMENTOS}

Os autores agradecem ao Conselho Nacional de Desenvolvimento Científico e Tecnológico (CNPq), Coordenação de Aperfeiçoamento de Pessoal de Nível Superior (CAPES) e à Fundação de Amparo à Pesquisa do Estado de Minas Gerais (FAPEMIG). 
AMABILI M.; FREGOLENT A., 1998, A Method to Identify Model Parameters and Gear Errors by Vibrations of a Spur Gear Pair, Journal of Sound and Vibration, Vol. 214, No. 2, pp. 339-357.

BRAUN, S., 1986, Mechanical Signature Analysis: Theory and Applications, 1.ed. Academic Press, London, 385p.

CHOI, F. K.; POLYSHCHUK, J. J. Z.; HANDSCUH, R. F.; TOWNSEND, D. P., 1996, Analysis of the Effects of Surface Pitting and Wear on the Vibration of a Gear Transmission System, Tribology International, Vol. 29, n. 1, pp. 77-83.

EDGAR, T. F., HIMMELBLAU, D. M., LASDON, L. S., 2001, Optimization of Chemical Process, Second Edition, McGraw-Hill Chemical Engineering Series, 651 pp.

HOWARD, I.; JIA, S.; WANG, J., 2001, The Dynamic Modeling of Spur Gear in Mesh Including Friction and a Crack, Mechanical System and Signal Processing, Vol. 5, pp. 438-449.

MACÁRIO, C. C. I., 2006, Utilização de filtros adaptativos para detecção de falhas em mancais de rolamentos, Dissertação de Mestrado - Universidade Federal de Uberlândia, Uberlândia, MG, Brasil.

MARQUES, P. A. C., 2003, Introdução à Filtragem Adaptativa. Instituto Superior de Engenharia de Lisboa, Apostila do curso de Processamento Digital de Sinal II - Engenharia Informática e de Computadores.

MENEGATTI, W. B., 1999, Identificação de Falhas em Caixas de Câmbios, Via Sinais de Vibração, Dissertação de Mestrado, Universidade Federal de Uberlândia, Uberlândia, MG, Brasil.

NUNES, M. A. A., 2006, Uma Metodologia para Quantificação da Contribuição Sonora de Fontes de Ruído Industrial no Meio Ambiente, Dissertação de Mestrado Universidade Federal de Uberlândia, Uberlândia, MG, Brasil.

OLIVEIRA FILHO, R. H, 2007, Uma Proposta de Ferramenta para Controle de Qualidade em Linha de Produção e Montagem de Câmbio Automotivos Utilizando Filtragem Adaptativa, Dissertação de Mestrado - Universidade Federal de Uberlândia, Uberlândia, MG, Brasil.

PENA, J. L. O., 2003, Um Estudo Sobre o Uso de Técnicas Não Tradicionais Aplicadas à Detecção de Defeitos de Fabricação em Câmbios Automotivos, Tese de Doutorado, Universidade Federal de Uberlândia, Uberlândia, MG, Brasil.

SANTANA, D.; RIBEIRO, C. R.; DUARTE, M. A. V.; CABACINHA, A., 1998, Um Procedimento Estatístico Para Identificação de Rumorosidades em Câmbios em Linhas de Montagem, Congresso SAE Brasil'98, São Paulo, Brasil.

SARAMAGO, S. F. P., 2003, Método de Otimização Randômica: Algoritmos Genéticos e Simulated Annealing, SBMAC, v. 6, 35p. 
STORN, R., PRICE, K.,1995, Differential Evolution: A Simple and Efficient Adaptive Scheme for Global Optimization over Continuous Spaces, Techinical Report TR 95-012, International Computer Science Institute, Berkeley.

VANDERPLAATS, G.N., 1999, Numerical Optimization Techniques for Engineering Design, VR D INC. Colorado Springs, CO, USA, Third Edition, 441p.

VELEX, P.; MAATAR, M., 1996, A Mathematical Model for Analyzing the Influence of Shape Deviations and Mounting Errors on Gear Dynamic Behavior, Mechanical Systems and Signal Processing. Vol. 191, No. 5, pp. 629-660.

WANG, W. J.; MACFADDEN, P. O., 1995, Decomposition of Gear Motion Signals and Its Application to Gearbox Diagnostics, ASME Journal of Vibration and Acoustics, Vol. 117, pp. 363-369.

WANG, W. Q.; ISMAIL, F.; GOLNARAGHI, M. F., 2001, Assessment of Gear Damage Monitoring Techniques Using Vibration Measurements, Mechanical Systems and Signal Processing. Vol. 15, No. 5, pp. 905-922. 